\title{
Antioxidant, antiradical, and antimicrobial activities of polysaccharides obtained by microwave-assisted extraction method: A review
}

Monirsadat Mirzadeh ${ }^{1}$, Mohammad Reza Arianejad ${ }^{2}$, Leila Khedmat ${ }^{3, *}$

${ }^{1}$ Metabolic Disease Research Center, Qazvin University of Medical Sciences, Qazvin, Iran ${ }^{2}$ Department of Food Science and Technology, Faculty of Nutrition, Tabriz University of Medical Sciences, Tabriz, Iran

${ }^{3}$ Health Management Research Center, Baqiyatallah University of Medical Sciences, Tehran, Iran

*Corresponding author's Email: lkhedmat@yahoo.com; Tel: +98-912-387-3622

\section{Highlights}

- Great bio-functionalities of polysaccharides extracted by microwave-assisted (MAE)

- The high capacity of polysaccharides to quench $\mathrm{DPPH}, \mathrm{OH}, \mathrm{NO}, \mathrm{ABTS}^{+}$, and $\mathrm{O}_{2}{ }^{--}$

- The dose-dependent reducing, chelating, and lipid peroxidation inhibition activities

- Uronic acids are the main constituents involved in the antioxidative properties

- Strong antibacterial, antifungal, and antiviral activities of MAE-polysaccharides

\begin{abstract}
The antioxidant and antimicrobial activities of polysaccharides extracted by microwave-assisted extraction (MAE) were reviewed. An ascending dose-dependent manner was found for the in vitro antioxidant (e.g., nitrite scavenging, phospho-molybdenum reduction, inhibition of lipid peroxidation (ILP), ferric reducing power, and ferrous metal ions chelating), and antiradical (against DPPH $\cdot, \mathrm{OH} \cdot$, ABTS $\cdot$, NO $\cdot$, and $\mathrm{O}_{2}{ }^{-}$) activities. There was a positive and significant correlation between ILP and erythrocyte hemolysis inhibition, showing the excellent antioxidative properties to prevent the risk of cell damage. These carbohydrate-based polymers in vivo could reduce malonaldehyde and protein carbonyls and increase stress-resistance-related enzymes such as catalase, superoxide dismutase, and glutathione peroxidase. They showed an effective bactericidal activity against a wide variety of gram-negative and gram-positive bacterial infections. The in vitro strong antifungal and antiviral activities of sulfated polysaccharides extracted by MAE were also diagnosed without any cytotoxicity effect. Therefore, these biomacromolecules might be used to develop functional foods and nutraceuticals.
\end{abstract}

